\title{
Eosinophilia, CTCAE
}

National Cancer Institute

\section{Source}

National Cancer Institute. Eosinophilia, CT CAE. NCI Thesaurus. Code C143190.

A disorder characterized by laboratory test results that indicate an increased number of eosinophils in the blood. 